\title{
Canadian
} Science Publishing

Canadian Journal of Physics

Revue canadienne de physique

\section{Cellular Automata Simulation for High Temperature Austenite Grain Growth based on Thermal Activation Theory and Curvature-Driven Mechanism}

\begin{tabular}{|r|l|}
\hline Journal: & Canadian Journal of Physics \\
\hline Manuscript ID & cjp-2016-0056.R3 \\
\hline Danuscript Type: & Article \\
\hline Complete List of Authors: & $\begin{array}{l}\text { Wang, Min; Huazhong University of Science and Technology, } \\
\text { Yin, Yajun; Huazhong University of Science and Technology } \\
\text { Zhou, Jianxin; Huazhong University of Science and Technology } \\
\text { Nan, Hai; AVIC Beijing Institute of Aeronautical Materials } \\
\text { Li, Wen; Huazhong University of Science and Technology } \\
\text { Wang, Tong; Huazhong University of Science and Technology }\end{array}$ \\
\hline Keyword: & $\begin{array}{l}\text { cellular automata, thermal activation, curvature-driven, grain growth, } \\
\text { computer simulation }\end{array}$ \\
\hline &
\end{tabular}

\section{SCHOLARONE ${ }^{\text {TM }}$}

Manuscripts 


\title{
Cellular Automata Simulation for High Temperature Austenite Grain Growth based on Thermal Activation Theory and Curvature-Driven Mechanism
}

\author{
Wang Min ${ }^{1}$, Yin Yajun ${ }^{1},{ }^{*}$ Zhou Jianxin ${ }^{1}$, Nan Hai ${ }^{2}$, Wang Tong ${ }^{1}$, Li Wen ${ }^{1}$ \\ 1, State Key Laboratory of Materials Processing and Die \& Mould Technology, Huazhong University of Science and Technology, Wuhan \\ 430074, China. 2, Beijing Institute of Aeronautical Materials, Aviation Industry of China, Beijing 100095, China \\ *Corresponding author: zhoujianxin@hust.edu.cn
}

\begin{abstract}
Based on the thermal activation theory and curvature-driven mechanism, a 2D cellular automaton model with different state transition rules was built. Validity of the model was proved by the shrinking of circular grains. Grain growth of high temperature austenite was simulated by this model, the morphology, grain size distribution; topological aspects and local kinetics of austenite grain growth were analyzed under different simulation time. Among the grains with different sides, the 6-sided grains are the most and 5-sided grains are the second most. The grains with more than 6 sides will grow and grains with less than 6 sides will shrink, while the 6-side grains will neither grow nor shrink. The kinetics of normal grain growth follows Burke equation and the growth exponent at different temperature and activation energy has been researched.
\end{abstract}

Key words: cellular automata, thermal activation, curvature-driven, grain growth, simulation

\section{Introduction}

Grain size is a reflection of the final microstructure characterization, and property of material is determined by the grain size. Microstructure control is one of the most important consider actions in process design. Traditionally, we have used optical microscopy, transmission electron microscopy and so on to study material microstructure. Shortcomings of the traditional methods are obvious: big workload and errors. Moreover, evolution of the microstructure can't be observed timely [1]. Cellular automaton (CA) model has been proposed to simulate the temporal evolution of microstructure. CA method is algorithms that describe the discrete spatial and/or temporal evolution of complex systems by applying local or global deterministic or probabilistic transformation rules to the sites of a lattice [2-3]. CA method has enjoyed a great improvement once put forward. Grain growth simulation by CA method can now be conducted in three dimensional areas [4-6] and shape of the lattice has developed from quadrangle to regular hexagon [7]. Moreover, CA method has been used in the simulation of austenite growing and transformation to ferrite. Golab R and Bachniak D have realized a sensitivity analysis of the developed complex micro scale austenite to ferrite phase transformation model based on CA [8]. In An D's paper, the preferential nucleation sites of austenite, the driving force of phase transformation coupled with thermodynamic parameters, solute partition at the ferrite/austenite interface, and carbon diffusion in both the ferrite and austenite phases are taken into consideration [9]. Lan Y J has provided a simple solution for the difficult moving boundary problem that governs the ferrite grain growth in his CA model [10]. Zheng C and Raabe D have study the microstructural transformation phenomena during the transformation that result from the interaction of primary recrystallization and phase transformation [11]. Also the post-dynamic transformation that takes place during the subsequent isothermal holding has also been investigated [12]. Chengwu Zheng and Dianzhong Li have developed a CA model to predict the microstructural evolution of the austenite recrystallization during multi-pass hot rolling of steel strip [13]. However, in the previous researches, state transition rules were mainly considering effects of the thermodynamic mechanism and the activation energy. Such as Liu Y [7] who has combined CA method with Monte Carlo method to simulate grain growth in two dimensional. Geiger [14] has put forward a cellular automaton method 
based on thermodynamics and successfully described the dynamics behavior, grain size distribution during the process of grain growths. And recently Janssens and Koenraad GF [15-16] have proposed a new three-dimensional, random grid based cellular automaton model for the simulation of microstructure evolution during recrystallization. They have described how the model can be linked to space and time coordinates and illustrated how theoretical analytical models describing grain boundary motion.

However, the above mentioned transition rules put their emphasis on the free energy change of the system. More specifically, if free energy of a cell is bigger than the energy to activate grain boundary migration, state of the cell will be changed. Results simulated by this model can explain some phenomena during grain growth. However, weakness of the model is obvious: the transition rules are simple. A common treatment is to combine a crystal plasticity finite-element model with a probabilistic cellular automaton [17-18]. Raabe $\mathrm{D}$ and his colleagues have done a remarkable work in this area. They have simulated the primary static recrystallization in aluminum by coupling of a crystal plasticity finite-element model with a probabilistic cellular automaton [19]. Besides, they have combined CA method with Monte Carlo method to study the polymer crystallization. Kinetics is formulated according to the Hoffman-Lauritzen secondary surface nucleation and growth theory for spherulite expansion and the local cell point switching probability is calculate by using a Monte Carlo step [20]. And recrystallization texture of an IF sheet steel under consideration of Zener pinning has been studied with a $2 \mathrm{D}$ cellular automaton [21]. Also a remarkable overview of constitutive laws, kinematics, homogenization and multiscale methods in crystal plasticity finite-element modeling have been done by them [22]. Other researchers, like Namin Xiao, have researched the dynamic recrystallization by coupling a cellular automation method with a topology deformation technique [23].

However, grain boundary curvature which will definitely affect the grain growth has been neglected in these models [24]. Recent researches have shown that grain boundary curvature is an assignable driving force for grain growth, and this view of point has been confirmed by a lot of experiments and theoretical analyses. Along with the clarity of the Curvature-Driven mechanism, transition rules based on the curvature-driven mechanism have been introduced to the newly developed CA models. Raghavan S [25] has built a new 2D CA model which can trace the grain boundary continuously and estimate grain boundary curvature accurately. Hua Fuan [26] has proposed a 2D cellular automata model for normal grain growth which based on the statistical theory of grain growth and the probabilistic rule [27-28]. In Hua's paper, the curvature-driven mechanism is explicitly introduced and a good linear relationship between the growth rate and the boundary curvature has been produced. However, his model has neglected the effects of temperature and thermal activation energy. In fact grain growth is a complicated physical process which is involved with many factors like kinetics and thermodynamics. Any cellular transformation rules based on a single mechanism can't accurately reflect the grain growth process.

Therefore, based on the thermal activation theory and curvature-driven mechanism, a 2D cellular automaton model with different state transition rules was built according to previous cellular automata models. Validity of the model was proved by the shrinking of circular grains. Microstructure evolution and the kinetics curves of the grain growth were obtained, moreover the grain growth processes were investigated at different temperatures and different activation energy respectively. Growth kinetics, grain size distribution have been analyzed. The results indicate that the grain growth of high temperature austenite is a process of big grain consuming small ones, and the stable grain should be a regular hexagon with rectilinear grain boundary. The growth exponent calculated by this model is 0.42 
at $1600 \mathrm{~K}$, which is a little smaller than the theoretical value of 0.5 . Additionally, the grain growth exponent has been calculated at different temperature and different activation energy. Obviously, influences of temperature and the activation energy on the growth exponent are small. Evolution of grains with different edges has been tracked. 4-sided grains and the 5-sided grains keep unchanged till the grains are gone. 6-sided grains stay the same. And sides number of grain with more than 6 sides keep changing during the grain growing process, and they have the chance to become an abnormal large grain size in the end.

\section{Theoretical Model of Normal Grain Growth}

As to the whole grain system, the driving force for grain growth is the difference of interfacial energy before and after the grain growth. However, for the micro process of a single grain growth, migration of the grain boundary is caused by the difference between grain interface curvatures. As to the large grain boundary migration, it is an atomic thermal activation transition process which is determined by the atomic energy barrier and the free energy difference. In the CA model, grain boundary energy is decided by the difference between grain orientations. When atoms leaped over the energy barrier and the transfer driving force between grains is different, then the grain boundary will migrate. According to the thermal activation theory in phase interface, the grain boundary migration rate can be expressed by the following term:

$$
v=M f
$$

In which, $M$ is the migration rate of large grain boundary, $f$ is the driving force of grain growth acting on the unit area. Expression of $M$ given as follows:

$$
M=\frac{\delta D_{o b} b}{K T} \exp \left(-\frac{Q_{b}}{R T}\right)
$$

Where $\delta$ is the grain boundary thickness of a specific material, $\mathrm{D}_{\mathrm{ob}}$ denotes the self-diffusion coefficient of grain boundary at absolute zero, $\mathrm{Q}_{\mathrm{b}}$ is the activation energy of diffusion, and $\mathrm{K}$ is the Boltzmann's constant. $\mathrm{b}$ is Burger's vector and $\mathrm{R}$ is the universal constant.

Expression of the driving force of grain growth given as follows:

$$
f_{i}=\gamma_{i} / r_{i}
$$

Where $\gamma_{i}$ represents the interfacial energy, which is determined by the difference between the grain orientations. The Read-Shockley equation [29] gives the calculation of the driving force:

$$
\gamma_{i}=\left\{\begin{array}{cc}
\gamma_{m} & \theta \geq 15^{\circ} \\
\gamma_{m} \frac{\theta_{i}}{\theta_{m}}\left(1-\ln \frac{\theta_{i}}{\theta_{m}}\right) & \theta<15^{\circ}
\end{array}\right.
$$

$\theta_{i}$ denotes the difference between the grain orientation, $\theta_{m}$ is the large angle grain boundary orientation

difference. And $\gamma_{m}$ is the large angle interfacial energy which can be calculated by the following expression ( $v$ is the Poisson's ratio of material, $\mu$ is the shear modulus):

$$
\gamma_{m}=\frac{\mu b \theta_{m}}{4 \pi(1-v)}
$$




\section{Cellular Automata Model}

The initial size of austenitic grains is relatively small in general. At the initial stage, grain boundary areas are relatively large, and system energy is relatively high. Along with the grain growth, the system energy is slowly lowered. Researches [30] have shown that the heating time, soaking time, heating rate and chemical composition can affect the grains growth process. In order to get a good simulation results, we should consider all these factors. In this paper, we have chosen a $2 \mathrm{D}$ area made up by $500 * 500$ rectangular cellular to simulate the grain growth. Four state transformation rules were established, effects of both the nearest and the second-nearest neighbor sites were employed. In the simulation, each cell contains two structure variables: (1) orientation of the cellular, in order to distinguish between different grains and a specific grain was represented by a randomly picked number between 1-180, at the same time the orientation number can be used to calculate the interfacial energy; (2) grain boundary variables, to mark whether a cellular cell was in the grain boundary. The state transition rules and the basic physical metallurgical principles of these rules have been discussed below:

\section{Transition Rule Based on Thermodynamic Mechanism}

As is known to all, moving of the grain boundary results in the grain growth. And boundary movement can be decomposed into migration of the atoms from one grain to another. However, only those atoms whose free energy is bigger enough to overcome the energy barrier can transit out of the boundary to the new grain. And adversely the atom will stay in the grain if the thermal activation energy is smaller than the energy barrier. In order to introduce to actual physical mechanism into the CA model, a transition rule based on this thermal activated process has been put forward. More specifically, the energy barrier in the proposed model is the boundary diffusion activation energy Qb. Only those atoms whose thermal activation energy is bigger than $\mathrm{Qb}$ have the opportunity to be transformed. For a certain cell, the transition probability is calculated by the following random probability formula:

$$
P=C \frac{T-T_{A c 1}}{T_{m}-T_{A c 1}} \exp \left(-\frac{Q_{b}}{R T}\right)
$$

Where $\mathrm{C}$ is a constant, when $\mathrm{T}=\mathrm{Tm}, \mathrm{P}=1$. We can calculate the constant $\mathrm{C}$ through this condition. And $\mathrm{R}$ is the universal gas constant, $\mathrm{T}$ is the absolute temperature and $\mathrm{Q}_{\mathrm{b}}$ is the boundary diffusion activation energy. $\mathrm{Tm}$ is the melting point of the material. $\mathrm{T}_{\mathrm{ACl}}$ is the austenitizing temperature. Obviously, this rule has considered the effects of temperature on the grain growth. Along with the increasing of temperature, the probability will increase. The physical meaning of the rule is that, at a specific temperature, only those cells whose thermal energy is bigger than the energy barrier have the chances to transform. Value of relevant parameters used in this paper can be found in table 1 :

Table 1: Value of parameter used in the paper

\section{Transition Rule Based on Curvature-Driven Mechanism}

Many experiments and theoretical researches have confirmed that when the grains grow to a steady state, the morphology of grain is hexagonal (shown in Fig.1). More specifically, the grain boundary is linear and angle between grain boundary turn out to be $120^{\circ}$. We can summarize the grain growth process as follow: firstly, the curving boundaries are moving to the center of curvature, in other words, the curving boundaries become straight. This process results in the reduction of surface area and interface energy. Secondly, when angle between three adjacent boundaries is not equal to $120^{\circ}$, the grain boundary will move in the direction of the grain boundary with the smallest angle (shown in Fig.1). 
Fig.1 Relationship between grain boundary curvature and grain shape

Generally speaking, the movement of grain boundary will end when angles between grain boundaries are all $120^{\circ}$. Based on the above regularity of normal grain growth, we have modeled a set of transition rules with considering the impact of the curvature-driven mechanism on the grain growth. Effects of both the nearest and next-nearest neighbors in the Moore configuration on the cellular transition have been discussed. A detailed description as follows:

Curvature-Driven Rule1: As to the consideration cell C5, if there are more than 5 neighbor cells whose state is the same (equal to $\mathrm{N}$ ) at a specific CA simulation step, the orientation state of core cell will be transformed to ' $\mathrm{N}$ ' (the transition situation has been shown in Fig.2). It can be expressed using the following formula:

$$
O_{t+\Delta t}^{C 5}=f\left(O_{t}^{C 1}, O_{t}^{C 2}, O_{t}^{C 3}, O_{t}^{C 4}, O_{t}^{C 6}, O_{t}^{C 7}, O_{t}^{C 8}, O_{t}^{C 9}\right)
$$

Fig.2 Diagram of the state translation based on curvature-driven rule 1

Curvature-Driven Rule2: This rule owns the second priority, and it mainly takes the effects of the 4 nearest neighbor cells into consideration. More specifically, if any three states of the nearest neighbor cells are identical (like equal to ' $\mathrm{N}$ '); then state of core cell will be transformed to ' $\mathrm{N}$ ' (The transition process depicted in Fig.3). It can be expressed using the following formula:

$$
O_{t+\Delta t}^{C 5}=g\left(O_{t}^{C 2}, O_{t}^{C 4}, O_{t}^{C 6}, O_{t}^{C 8}\right)
$$

Fig.3 Diagram of the state translation based on curvature-driven rule 2

Curvature-Driven Rule3: This rule shares the third priority, and the transition probability of the core cell is up to the orientation of the next-nearest cells. More specifically, if any three states of these cells are of the same orientation like ' $\mathrm{N}$ ', orientation of the core cell will be transformed (shown in Fig.4). It can be expressed using the following formula:

$$
O_{t+\Delta t}^{C 5}=h\left(O_{t}^{C 1}, O_{t}^{C 3}, O_{t}^{C 7}, O_{t}^{C 9}\right)
$$

Fig.4 Diagram of the state translation based on curvature-driven rule 3

\section{Transition Rule Based on Energy Mechanism}

As mentioned before, the interfacial energy can somehow be determined by the grain boundary curvature. Thus the boundary curvature changes can be converted to calculate the change of interfacial energy. If the above curvature driven mechanism fail to meet, then a transition probability has been introduced to determine the transition of the core cell. And the transition probability of core cell is calculated by the lowest energy principle. Isotropy of interfacial energy has been assumed, interfacial energy of the cellular cell i can be calculated by Hamiltonian function:

$$
E_{i}=J \sum_{k}^{R}\left(1-\delta C_{i} C_{k}\right)
$$

Where $\mathrm{J}$ is a measurement for grain boundary energy, and its value is 1 in this paper, $\delta$ is Kronecker symbol, $\mathrm{k}$ is the kth neighbor to cell $\mathrm{i}, \mathrm{R}$ is the total neighbor number of the cell $\mathrm{i}$, and its value is 8 in two dimensional simulation area. $\mathrm{C}_{\mathrm{i}}$ and $\mathrm{C}_{\mathrm{k}}$ are the orientation of cell $\mathrm{i}$ and $\mathrm{k}$ respectively. The state of cell C5 randomly translates to an arbitrary neighbor (C1,C2,C3,C4,C6,C7,C8,C9 seen in Fig.5). The energy change of cell $\mathrm{C} 5$ after its transition is calculated by the following equation:

$$
\Delta E_{i \rightarrow j}=E_{j}-E_{i}=J \sum_{k}^{R}\left(1-\delta C_{i} C_{k}\right)-J \sum_{k}^{R}\left(1-\delta C_{j} C_{k}\right)
$$


If $\Delta E_{i \rightarrow j}<0$, the transition probability is equal to 1 ; and C5 cell can't be translated if $\Delta E_{i \rightarrow j} \geq 0$.

Fig.5 Diagram of the state translation based on energy mechanism

The simulation model used in this paper has considered the effects of both the curvature driven mechanism and thermodynamic driving mechanism. Four state transformation rules have been established based on these effects, and the simulation process can be done in the following sequence (shown in Fig.6). First of all, initialize parameters used in this paper, and generate the initial grains for the simulation. As we all know, grains growth is a process of grain boundary moving. Movement of grain boundaries involves the transfer of a group of atoms from one grain to a neighbor across the grain boundary. For a certain cell, a random number between 0 and 1 is generated, if the number is smaller than the transition probability $\mathrm{P}$, then the curvature-driven mechanisms will be checked orderly. In fact, the proposed curvature-driven mechanisms are the effects of the neighbor cells state on the transition of the present cell. And the smaller distance between cells, the larger effects on the transition of present cell. In other words, the curvature-driven Rule 1 will be given much more priority than Rule 2, and Rule 2 will be given precedence compared with Rule 3. If conditions of curvature-driven Rule 1 have been satisfied, state of the present cell will be changed. If Rule 1 fails to describe the situation, then Rule 2 will be concerned. And Rule 3 will be considered only in the case that Rule 1 and Rule 2 both do not work. If all the three transition rules do not work, then the energy rule will work: a neighbor cell will be randomly picked to calculate the changes of the system energy according to the mentioned equation. State of the present cell will be changed if the system energy becomes smaller. If the system energy becomes bigger, the present cell remains its original state. In each CA simulation step, the whole cells will be checked.

Fig.6 The flowchart of the CA model to do the grain growth simulation

\section{Validation of the Presented Model}

The validity of the model developed in this paper was proved by the shrinking of circular grains. If the circular grain is shrinking in an ideal condition, according to the normal grain growth model mentioned above, the grain growth velocity can be got in the following expression:

$$
v=\frac{d r}{d t}=-\frac{M \gamma_{i}}{r_{i}}
$$

A simplified form of the integration of the above expression as follows:

$$
r^{2}-r_{0}^{2}=-k t
$$

Where $\mathrm{k}$ is a function of grain boundary mobility $M$ and interfacial energy $\gamma_{i}, r_{0}$ denotes the initial radius of the grain, $t$ represents the time consumed during the grain growth. From the derived equation, we can make the conclusion that radius squared is proportional to the grain growth time. The shrinking process of a circular grain has been simulated based on the proposed model, results can be seen in Fig.7. As can be found in the picture, along with the increasing of CAS, the circular grain is shrinking gradually with the help of the curvature-driven force. The newly formed grain keeps circular basically. Local fluctuations and deviation of the grain shape are due to the anisotropy of the cellular grids and thermal activation mechanism. Fig.8 depicts the kinetic curve during the circular grain shrinking process; it shows that radius squared is proportional to the CAS. And this phenomenon is consistent with the theoretical one. Accuracy of the model proposed in this paper can be proved by comparing the 
simulation results and the theoretical one.

Fig.7 Shapes of circular grains at different time steps in shrinking

Fig.8 Kinetics curves of circular grain shrinking

\section{Simulation Results and Discussions}

\section{Microstructure Simulation Results}

The initial orientations of cellular in the simulation area were randomly set to $R(1 \leq R \leq 180)$, and the initial grains are the same at different simulation temperature. In each CAS, all the cells will be checked in accordance with the above four transition rules. The microstructure evolution process at different temperature can be seen in Fig.9. And Fig.10 shows the microstructure evolution process at different activation energy. As shown in these figures. The homogeneously distributed grains generally have a polygonal shape in the current simulation [31]. More specifically, the average grain size in the simulation scale increases along with the simulation time at a certain temperature, and number of grains becomes fewer. At the same simulation step, the higher temperature gives a larger grain size. As can be seen from the Fig.10, at a fixed temperature, it's easier for the grains to grow up when the activation energy is smaller. This is because if the activation energy is small, it's easy for the atoms located at the grain boundary to overcome the energy barrier to transit, the grain boundary moves along with the atoms transition and result in the grains growth. Also, the bigger grains become much bigger while the small ones become much smaller. As can be seen in the pictures, there are some small grains around the bigger one. And we can make the conclusion that grains growth is a process in which the big grains eat up the small. Moreover, along with the increasing of CAS, number of isometric grains increases. And grain boundary tends to be straight. Another obvious characteristic is that grain growth is a continuous and uniform process. In other words, there didn't appear grains in relatively large size. These results are consistent with actual laws.

Fig.9 Microstructure evolution at different CAS different temperature

Fig.10 Microstructure evolution at different CAS different activation energy

\section{Grain size distribution}

As is known to all, grain size distribution is an important index for measuring the uniformity of microstructures. Changing of the relative grain size (Relative grain size is denoted by R/Rm, in which $\mathrm{R}$ represents the size of different grain and $\mathrm{Rm}$ denotes the average grain size) distribution along with the CAS has been depicted in Fig.11. As shown in the figure below, it is easy to find that grain size distribution is not very stable at the beginning stage of the simulation. Along with the increasing of CAS, distribution of the relative grain size conforms to the logarithmic normal distribution. Moreover, the grain size distribution is almost time-invariable during normal grain growth. We can also find that along with the grain growth, most of the grain size tends to be equal to the average grain size in the microstructure. In other words, the maximum frequency is at $\mathrm{R} / \mathrm{Rm}=1.0$. The distribution frequency approaches to zero when $\mathrm{R} / \mathrm{Rm}$ tends to be zero. On the other hand, there is an apparent cutoff at $\mathrm{R} / \mathrm{Rm}=2.4$. This agrees well with the results of Fan D's work [32].

Fig.11 Relative grain size distributions at different CAS

\section{Dynamics of Grain growth}

Beck [33] has established the relationship between the average grain sizes with the grain growth time in 1947. The grain growth kinetics can be expressed as the following form based on the experimental results:

$$
R_{m}=B t^{n}
$$


In the above expression, $\mathrm{Rm}$ represents the average grain size, and $\mathrm{n}$ represents the growth exponent. Previous researches have shown that the growth exponent is always the most controversial point. Results from the previous researchers are different which are based on different experiments and simulation models [32-34]. In a practical application, many studies have chosen a theoretical value of $n$ equal to $0.5[14,35]$. The logarithm form of the above expression has been taken as follow:

$$
\ln R_{m}=\ln B+n \ln t
$$

Obviously, the grain growth exponent can be calculated by calculating the slope of the lnRm-lnt line. Kinetic curve of grain growth and its logarithmic form at the situation which temperature is $1600 \mathrm{~K}$ can be found in Fig. 12.

Fig.12 Relation between average grain size and time during grain growth

we can find that the Rm-t line shaped like a ' $\mathrm{S}$ ', that is to say, size of the grain become bigger along with the CAS. This phenomenon can be well explained by Beck's law. The grain growth exponent $n$ calculated by the right picture is 0.42 . The calculated grain growth exponent $n$ is a little smaller than the theoretical value 0.5 . Researches have shown that the growth exponent is closely related to the kinetic parameters like temperature, grain boundary mobility, activation energy of diffusion and so on. Grain growth exponent can be less than 0.5 even in the case of pure metals, where the influence of precipitates and impurities are almost inexistent [36-38]. Accordingly, the grain growth exponent in pure iron varies from 0.25 to 0.5 with increasing temperature and reaches 0.5 at high temperature. Therefore, difference of parameters used in the numerical simulation experiment will inevitably lead to the difference of the calculated growth exponent compared with the theoretical value. Moreover, as can be seen in the right picture of Fig.12, at the beginning CA simulation steps, the initial microstructure show great instability, the grain growth is fast and disordered; this is because the free energy of the system is relatively high in the initial stage. Growth exponent is affected by the instability of the early stage of grain growing. While in the later stage of grain growth, grains growth are more smoothly. So kinetic curves of grain growth at different temperature and different activation energy have been researched and results can be found in Fig.13. We have removed the initial stage of the grains growth process in order to eliminate the effects of the instability of early stage. The left one in Fig.13 is the kinetic curve at different temperature, and the right one shows the kinetic curves at different activation energy.

Fig.13 Relation between average grain size and CAS at different temperature and activation energy (A) different temperature (B) different activation energy

From the above picture, we can find that the average grain size becomes bigger along with the increase of temperature. The growth exponents at different temperature are: $0.45,0.45,0.46$, and 0.46 . Kinetic curve of grain growth at different activation energy can be found in the right picture, it's easy to find that the smaller the activation energy and bigger the average grain size. This is because the smaller the activation energy, the bigger chance for the atoms to jump out from the grain boundary. Three growth exponents at different activation energy are: $0.46,0.46$ and 0.47 . From this comparison results, we can make the conclusion that temperature and activation energy can affect the average grains size but their influences on the grain growth exponent are limited.

\section{Grain growth Topology}

Fig.14 (A) shows the distribution of grain with different grain side (definition of grain side here is the number of neighbor grains around the specific grain) at different simulation step at $1600 \mathrm{~K}$. From the picture, we can find that distribution of the grain side conforms to the logarithmic normal distribution. 
More specifically, number of the grains with less than 2 sides and number of grains with more than 10 sides are relatively fewer in the whole system. And grains with 6 sides and 5 sides share the maximum frequency. The figure also indicates the distributing characteristics have nothing to do with the simulation time and temperature. These agree well with the results of Fan [32].

Fig.14 (A) Distribution of grains with different sides, (B) Growth kinetics of grains with different sides Mullins [39] has studied the relationship between grain area changing and its topological structure. In his paper, he has made the conclusion that grains with more than 6 sides tend to grow bigger, while grains with less than 6 sides tend to be consumed by other grains. And the 6 sided grains keep unchanged during the evolution process. Fig.14 (B) shows the grains growth kinetics. As can be seen in the picture, the growing rate of grains with more than 6 sides is smaller than the shrinking rate of grains with less than 6 sides. These are in good agreement with the expressed by the Mullins equation.

\section{Fig.15 Evolution of grain with different edges}

As discussed in the above paragraph, grains with 4-6 sides have the higher chance to form during the grain growth. So the topological evolution of the 4 sided grain, 5 sided grain and 6 sided grain have been analyzed in detail (Seen in Fig.15). As can be seen, along with the grain growth, sides of the 4 sided grain and the 5 sided grain keep unchanged till the grain is gone. It's not difficult to find that sides of the 6 sided grains stay the same. These features correspond to the experimental results and the simulation results done by other researchers [40-41]. From the above analysis, number of grains with more than 6 edges is relatively small in the whole simulation area, but they do exist, Fig. 16 has given a vividly description of the growing process of grain with more than 6 edges.

\section{Fig.16 Evolution of grain with more than 6 edges}

As can be seen in Fig.16, grain with more 6 edges is relatively bigger than the other grains. What's more, the edges number of grain with more than 6 edges change constantly along with the evolution of grain growth. This is because the relatively small grains were consumed by the neighbor bigger grains. On the other side, once the grain with more than 6 edges is formed, it's very difficult to be swallowed up by the other grains in the following grains growth process. And this results in the abnormally large grain which will appear in actual process in the end.

\section{Conclusions}

Curvature-driven mechanism was introduced to simulate grain growth in this paper. The mechanism was introduced by considering effects of neighboring grids on the transformation probability of present grid. Three transition rules with different priority have proved that the smaller distance between present cell and neighboring cell, the bigger influences on the state transformation probability. And this has provided guidance for the construction of the proposed model in three dimensional.

In full consideration of effects of the basic physical metallurgical principles acting in grain growth, we have combined the thermodynamic driving mechanism with curvature-driven mechanism. Process of grain growth has been simulated. The proposed model has been validated through the shrinking of the circular grain. Moreover, the morphology, grain size distribution, topological aspects and local kinetics of austenite grain growth were analyzed, and simulation results tally with the theoretical results.

Growth exponent at $1600 \mathrm{~K}$ temperature is equal 0.42 which is a little smaller than the theoretical value, which is due to the instability of the initial stage of grain growth. After stripping out the initial grains growth stage, grains growth exponent at different temperature and activation energy have been researched, and the grain growth exponent is around 0.47. Effects of temperature and activation energy on grain growth exponent are relatively small based on the comparison results. 


\section{Acknowledgments}

This research was financially supported by National Natural Science Foundation of China (No.51305149), and National Science \& Technology Key Projects of Numerical Control (2012ZX04012-011).

\section{References}

1. X. Li, Q. Zhou, M. Chen and X. Wang, Materials for Mechanical Engineering 7, 26 (2012). doi:

2. H. W. Hesselbarth and I. R. Göbel, Acta Metallurgica et Materialia 39 (9), 2135 (1991). doi:

3. D. Raabe, Annu. Rev. Mater. Res. 32 (1), 53 (2002). doi:

4. H. L. Ding, Y. Z. He, L. F. Liu and W. J. Ding, J. Cryst. Growth 293 (2), 489 (2006). doi: 10.1016/j.jcrysgro.2006.05.060

5. D. Raabe, Philosophical Magazine A 79 (10), 2339 (1999). doi:

6. Y. Vertyagina and M. Mahfouf, J. Mater. Sci. 50 (2), 745 (2015). doi:

7. Y. Liu, T. Baudin and R. Penelle, Scripta Mater. 34 (11), 1679 (1996). doi:

8. R. Golab, D. Bachniak, K. Bzowski and L. Madej, Applied Mathematics 4 (11), 1531 (2013). doi:

9. D. An, S. Pan, L. Huang, T. Dai, B. Krakauer and M. Zhu, ISIJ Int. 54 (2), 422 (2014). doi:

10. Y. J. Lan, D. Z. Li, C. J. Huang and Y. Y. Li, Model. Simul. Mater. Sc. 12 (4), 719 (2004). doi:

11. C. Zheng and D. Raabe, Acta Mater. $61 \quad$ (14), 5504 (2013). doi: 10.1016/j.actamat.2013.05.040

12. C. Zheng, D. Raabe and D. Li, Acta Mater. 60 (12), 4768 (2012). doi: 10.1016/j.actamat.2012.06.007

13. C. Zheng, N. Xiao, D. Li and Y. Li, Comp. Mater. Sci. 44 (2), 507 (2008). doi: 10.1016/j.commatsci.2008.04.010

14. J. Geiger, A. Roósz and P. Barkóczy, Acta Mater. 49 (4), 623 (2001). doi: 10.1016/S1359-6454(00)00352-9

15. K. G. Janssens, Model. Simul. Mater. Sc. 11 (2), 157 (2003). doi:

16. K. G. F. Janssens, Math. Comput. Simulat. 80 (7), 1361 (2010). doi: 10.1016/j.matcom.2009.02.011

17. F. Han, B. Tang, H. Kou, L. Cheng, J. Li and Y. Feng, J. Mater. Sci. 49 (8), 3253 (2014). doi:

18. H. Won Lee and Y. Im, Int. J. Mech. Sci. 52 (10), 1277 (2010). doi: 10.1016/j.ijmecsci.2010.06.003

19. D. Raabe and R. C. Becker, Model. Simul. Mater. Sc. 8 (4), 445 (2000). doi:

20. D. Raabe, Acta Mater. 52 (9), 2653 (2004). doi: 10.1016/j.actamat.2004.02.013

21. D. Raabe and L. Hantcherli, Comp. Mater. Sci. 34 (4), 299 (2005). doi: 10.1016/j.commatsci.2004.12.067

22. F. Roters, P. Eisenlohr, L. Hantcherli, D. D. Tjahjanto, T. R. Bieler and D. Raabe, Acta Mater. 58 (4), 1152 (2010). doi: 10.1016/j.actamat.2009.10.058

23. N. Xiao, C. Zheng, D. Li and Y. Li, Comp. Mater. Sci. 41 (3), 366 (2008). doi: 10.1016/j.commatsci.2007.04.021

24. A. D. Rollett and D. Raabe, Comp. Mater. Sci. 21 (1), 69 (2001). doi: 10.1016/S0927-0256(00)00216-0 
25. S. Raghavan and S. S. Sahay, Comp. Mater. Sci. 46 (1), 92 (2009). doi: 10.1016/j.commatsci.2009.01.028

26. F. Hua, Y. Yang, D. Guo, W. Tong and Z. Hu, ACTA METALLURGICA SINICA-CHINESE EDITION- 40, 1210 (2004). doi:

27. G. Abbruzzese and K. Lücke, Acta Metallurgica 34 (5), 905 (1986). doi:

28. H. Eichelkraut, G. Abbruzzese and K. Lücke, Acta Metallurgica 36 (1), 55 (1988). doi:

29. W. T. Read and W. Shockley, Physical Review 78 (3), 275 (1950). doi:

30. C. Zhongxi, Metallurgy and Heat Treatment Theory. (China Machine Press, Beijing, 2004).

31. F. Chen, Z. Cui, J. Liu, W. Chen and S. Chen, Materials Science and Engineering: A 527 (2122), 5539 (2010). doi: 10.1016/j.msea.2010.05.021

32. D. Fan, C. Geng and L. Chen, Acta Mater. 45 (3), 1115 (1997). doi:

33. P. A. Beck, M. L. Holzworth and H. Hu, Physical Review 73 (5), 526 (1948). doi:

34. V. Petrovic and M. M. Ristic, Metallography 13 (4), 319 (1980). doi:

35. E. A. Grey and G. T. Higgins, Acta Metallurgica 21 (4), 309 (1973). doi:

36. H. Hu and B. B. Rath, Metallurgical Transactions 1 (11), 3181 (1970). doi:

37. R. A. Vandermeer and H. Hu, Acta Metallurgica et Materialia 42 (9), 3071 (1994). doi:

38. H. H, Can. Metall. Quart. 1 (13), 275 (1974). doi:

39. W. W. Mullins, J. Appl. Phys. 27 (8), 900 (1956). doi:

40. M. Palmer, K. Rajan, M. Glicksman, V. Fradkov and J. Nordberg, Metallurgical and Materials Transactions A 26 (5), 1061 (1995). doi:

41. Y. He, H. Ding, L. Liu and K. Shin, Materials Science and Engineering: A 429 (1-2), 236 (2006). doi: 10.1016/j.msea.2006.05.070 
Table 1: Value of parameter used in the paper

\begin{tabular}{ccc}
\hline Parameters & Values & Units \\
\hline $\mathrm{Q}_{\mathrm{b}}$ & 159 & $\mathrm{~kJ} / \mathrm{mol}$ \\
$\mathrm{b}$ & $2.58 \times 10^{-10}$ & $\mathrm{~m}$ \\
$\mathrm{D}_{0 \mathrm{~b}}$ & 7.5 & $\mathrm{~m}^{3} / \mathrm{s}$ \\
$\mathrm{T}_{\mathrm{m}}$ & 1810 & $\mathrm{~K}$ \\
$\mathrm{~T}_{\mathrm{AC} 1}$ & 1000 & $\mathrm{~K}$ \\
$\mathrm{C}$ & 36297 & $/$ \\
\hline
\end{tabular}

Fig.1 Relationship between grain boundary curvature and grain shape
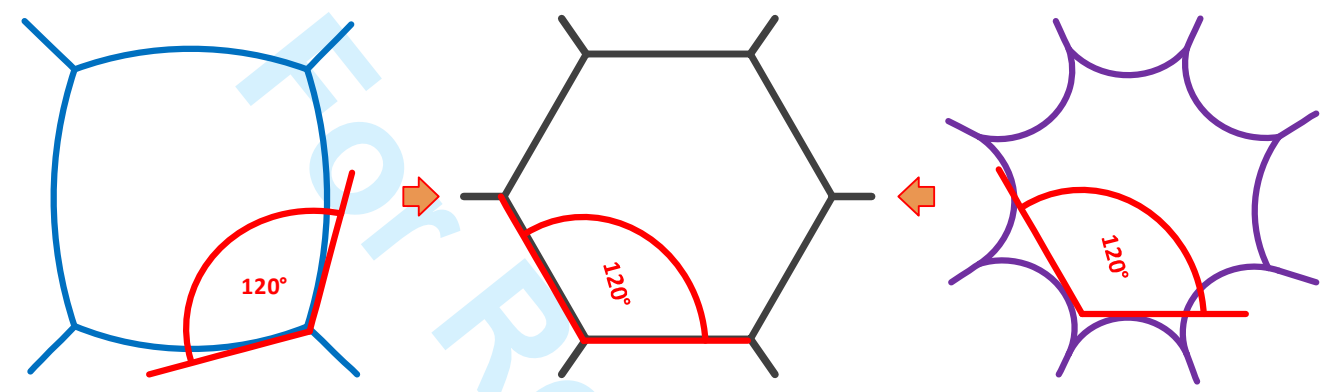

Fig.2 Diagram of the state translation based on curvature-driven rule 1

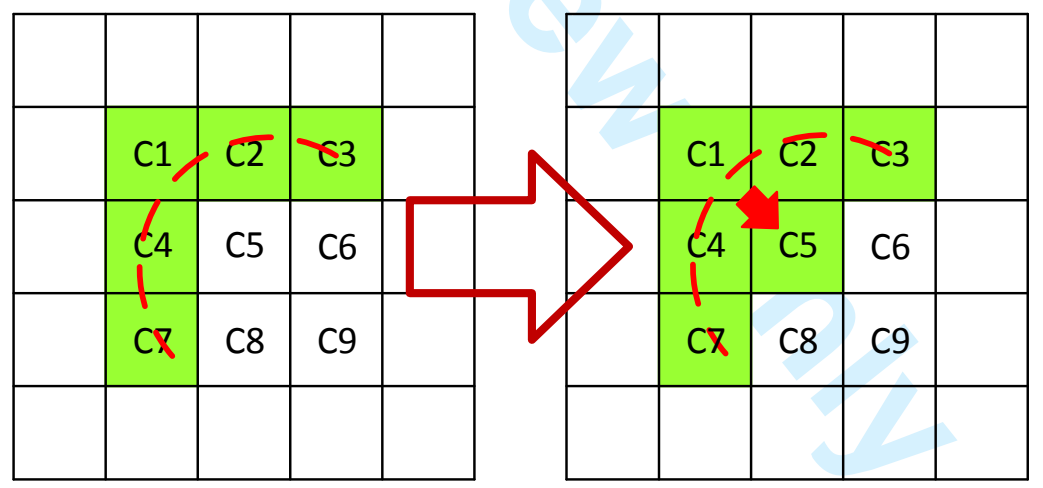

Fig.3 Diagram of the state translation based on curvature-driven rule 2 


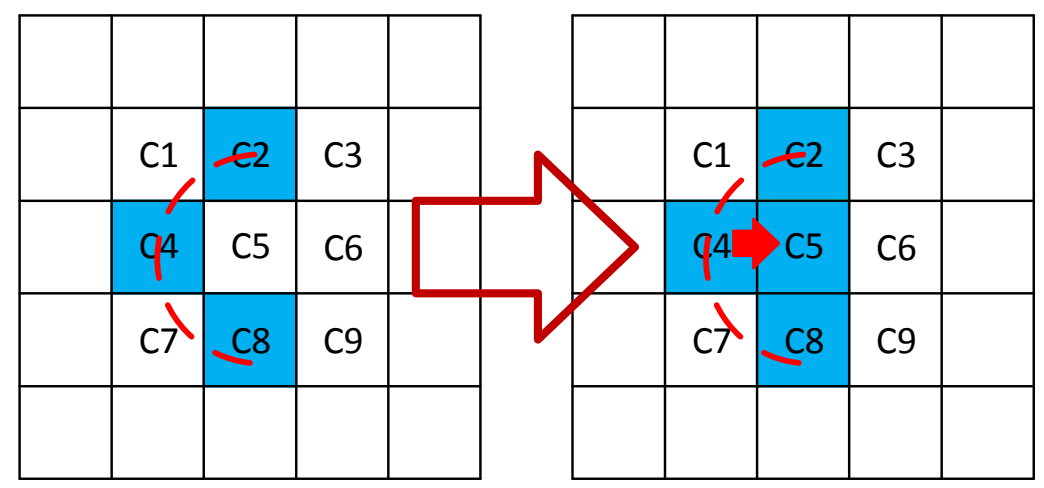

Fig.4 Diagram of the state translation based on curvature-driven rule 3

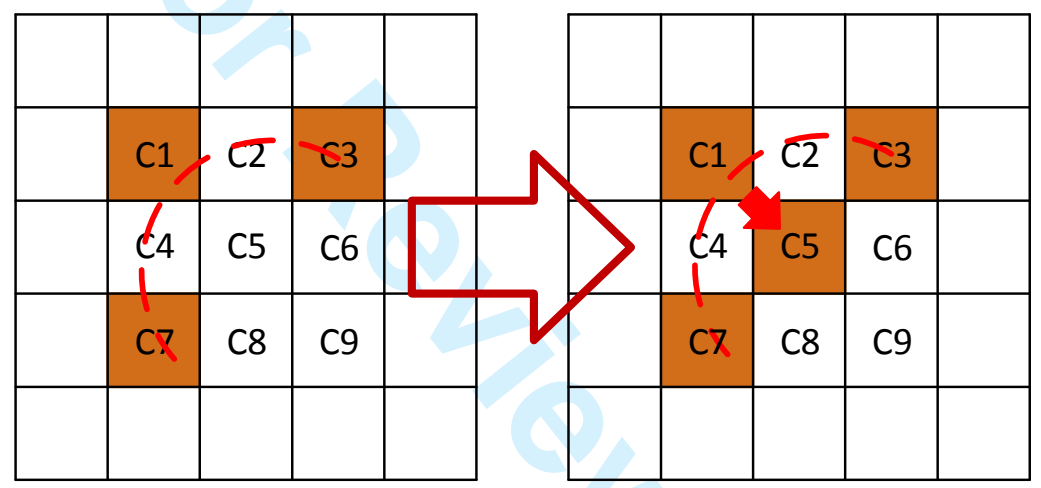

Fig.5 Diagram of the state translation based on energy mechanism

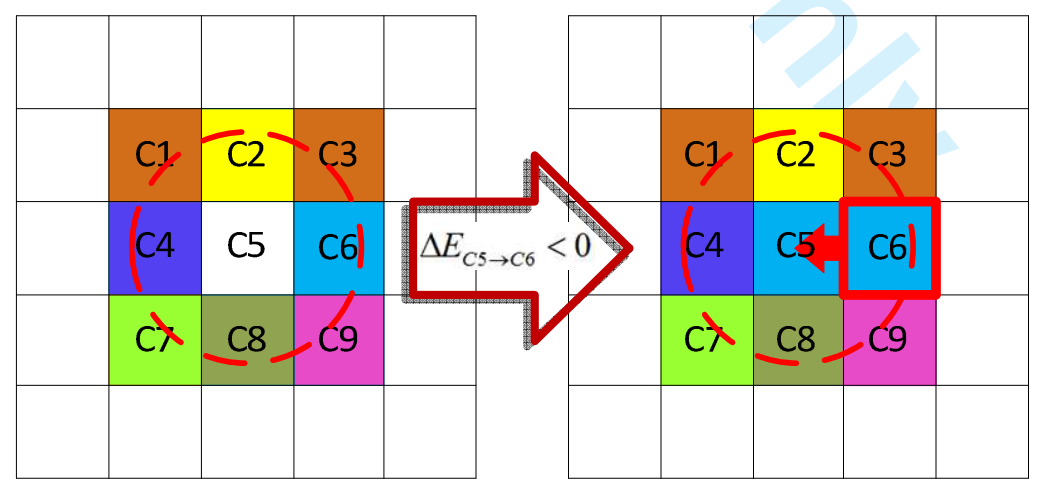


Fig.6 The flowchart of the CA model to do the grain growth simulation

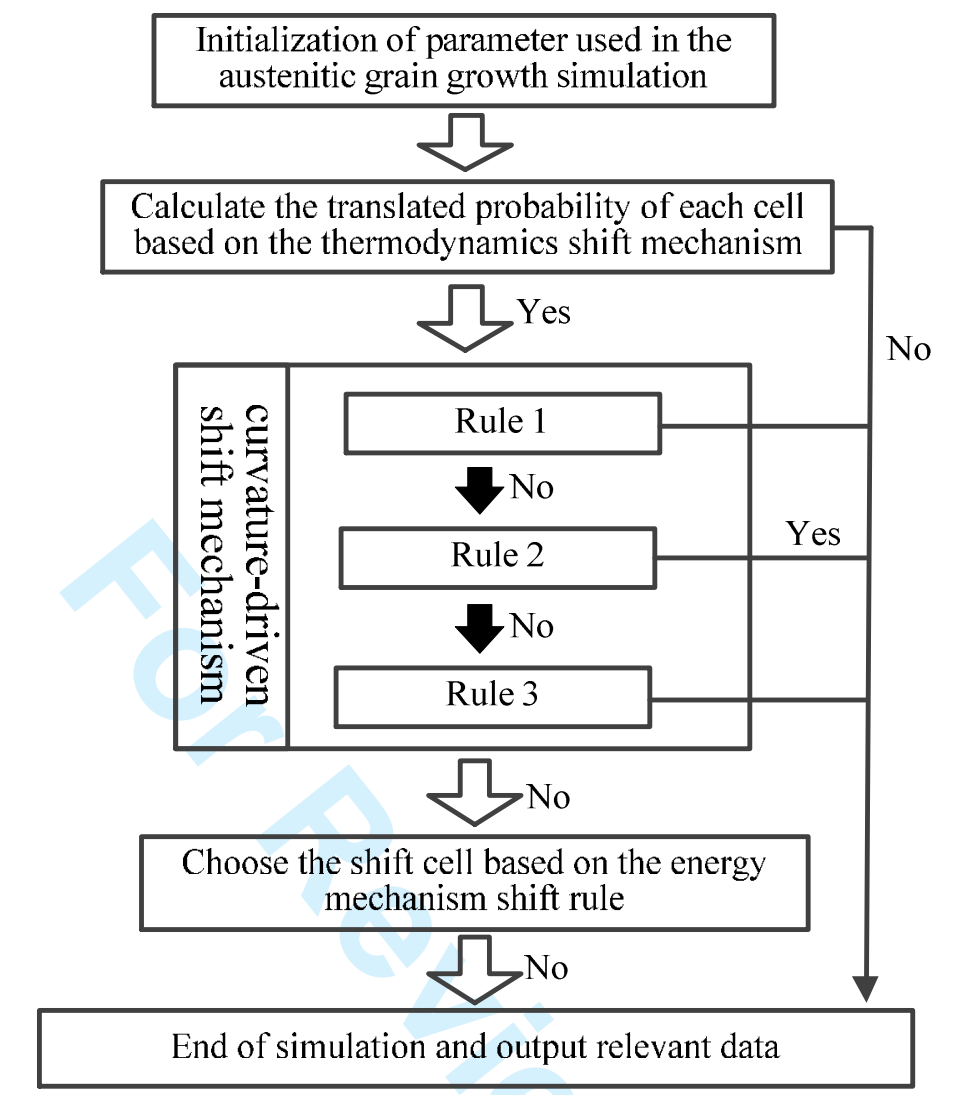

Fig.7 Shapes of circular grains at different time steps in shrinking

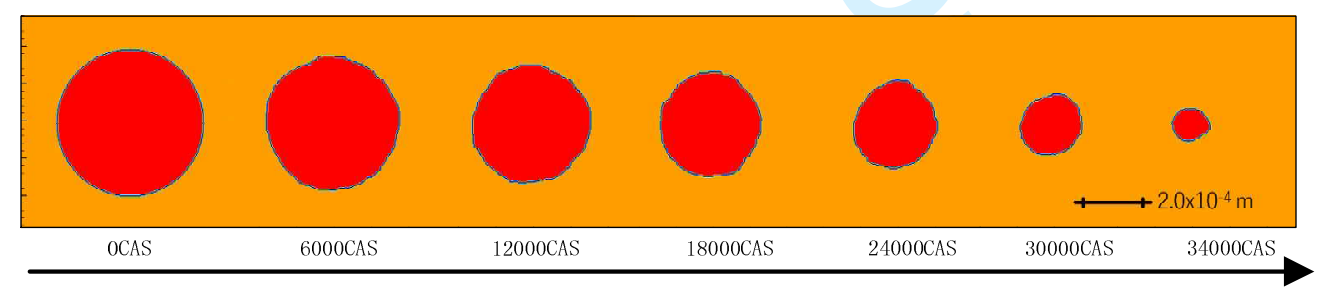


Fig.8 Kinetics curves of circular grain shrinking

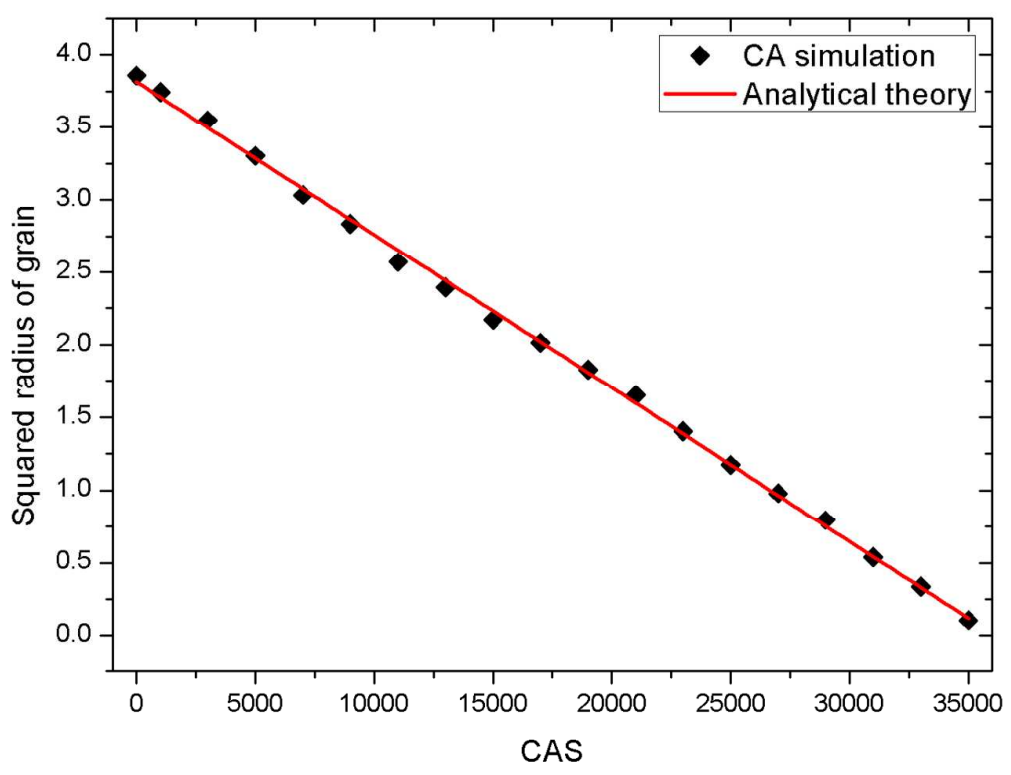

Fig.9 Microstructure evolution at different CAS different temperature
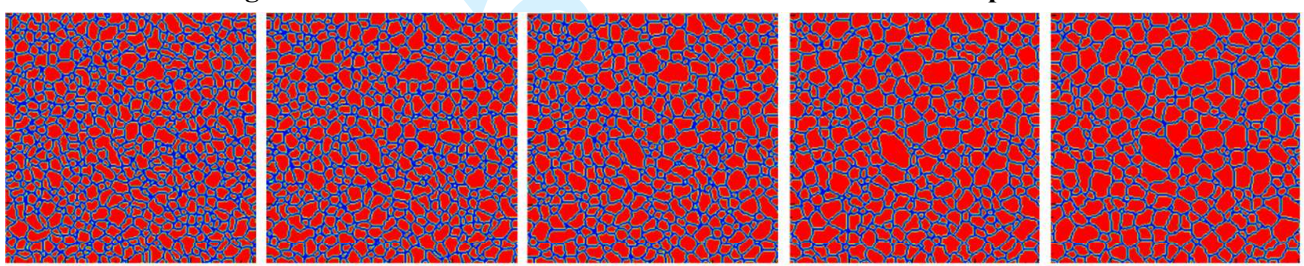

(A) $1300 \mathrm{~K}$
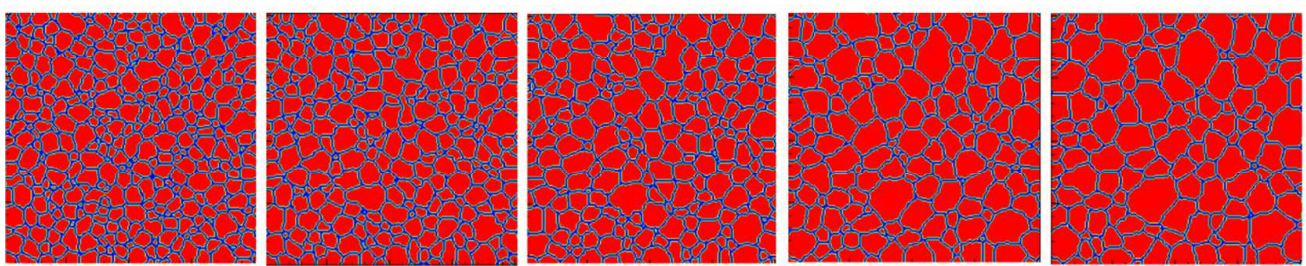

(B) $1400 \mathrm{~K}$
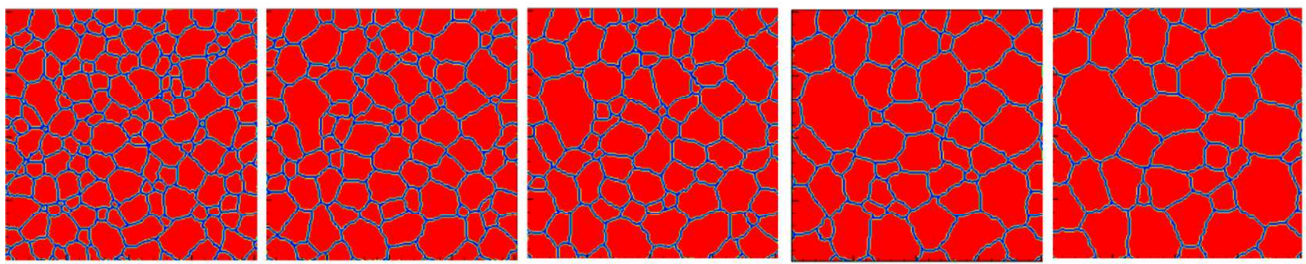

(C) $1500 \mathrm{~K}$
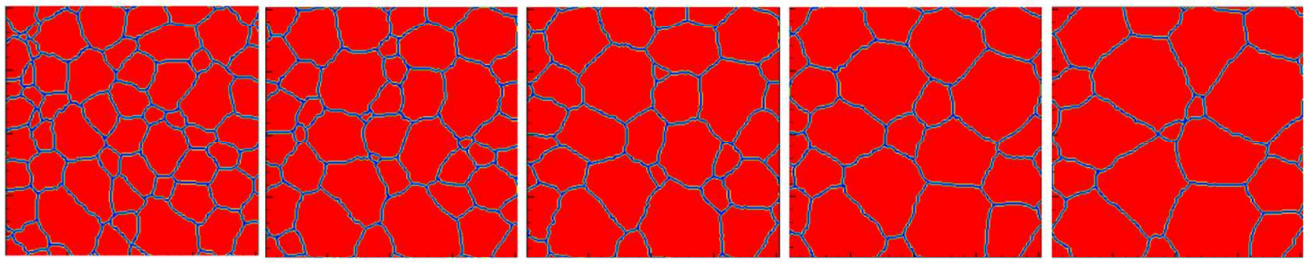

(D) $1600 \mathrm{~K}$

4000CAS

6000CAS

8000CAS

12000CAS

16000CAS 
Fig.10 Microstructure evolution at different CAS different activation energy
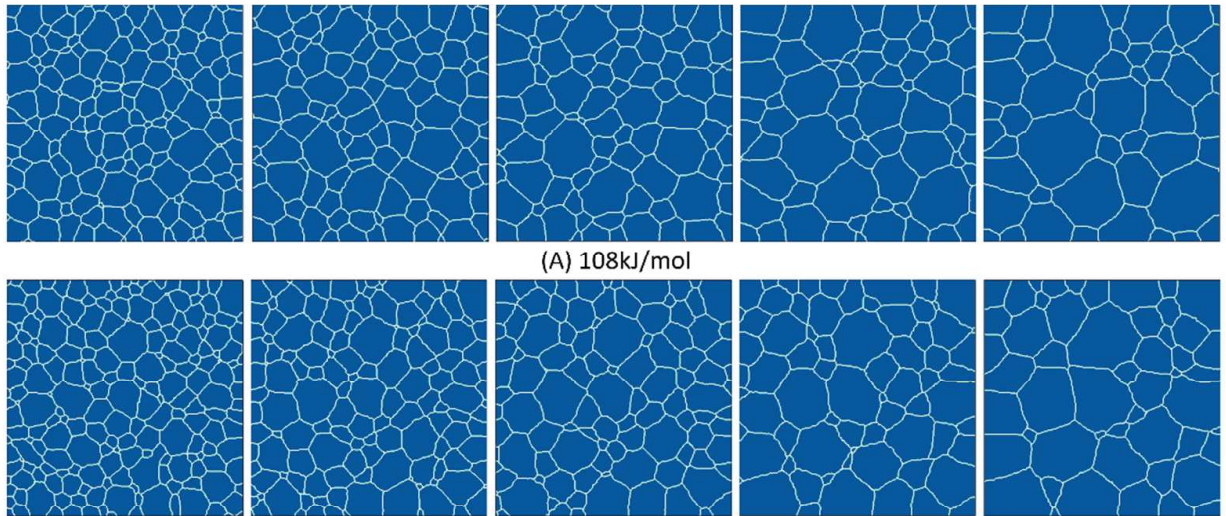

(A) $108 \mathrm{~kJ} / \mathrm{mol}$
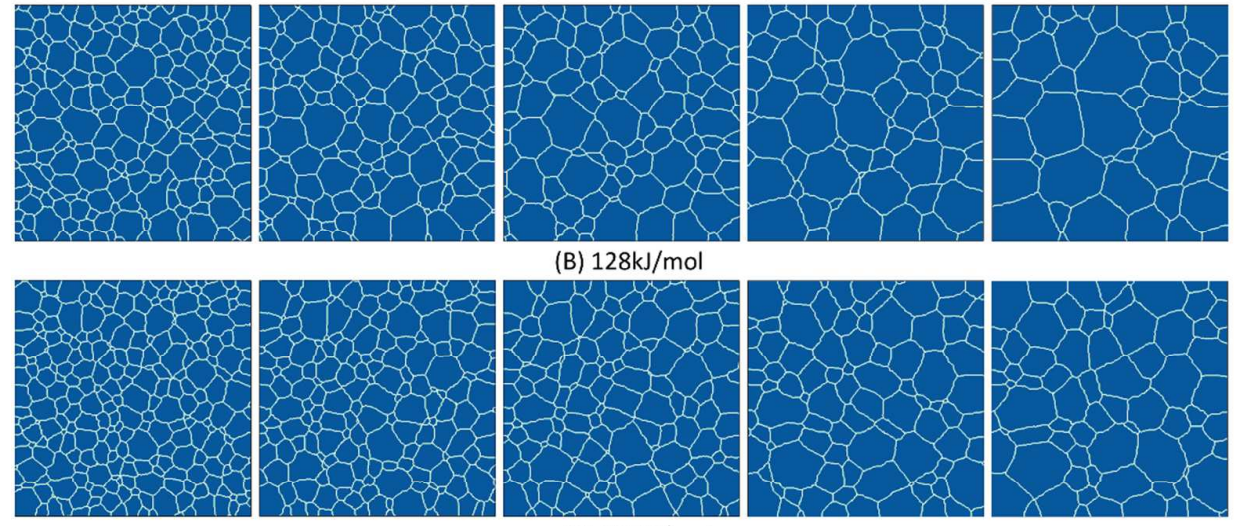

(B) $128 \mathrm{~kJ} / \mathrm{mol}$
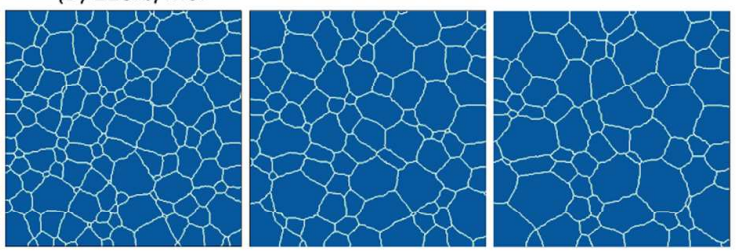

4000CAS

6000CAS

8000CAS

12000CAS

16000CAS

Fig.11 Relative grain size distributions at different CAS

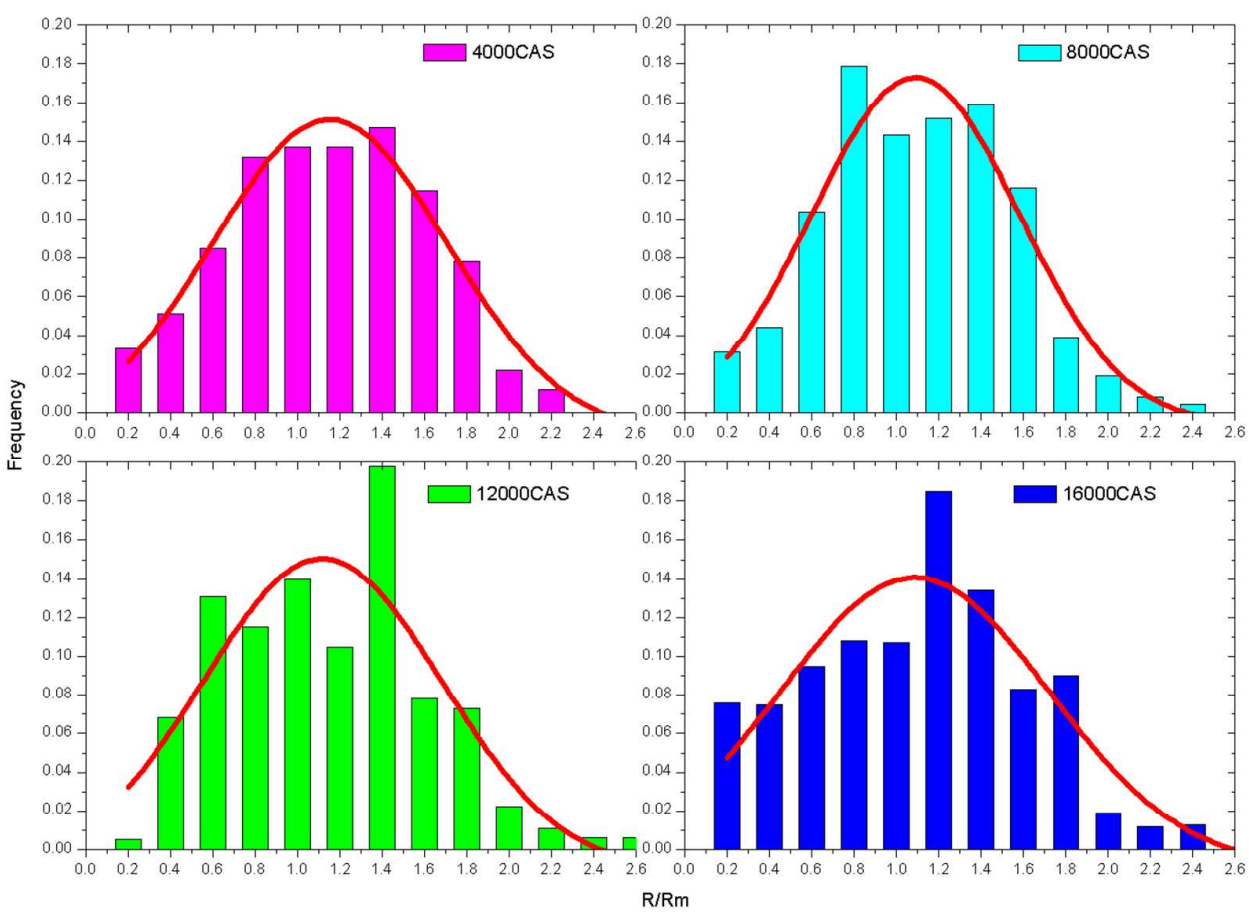


Fig.12 Relation between average grain size and time during grain growth

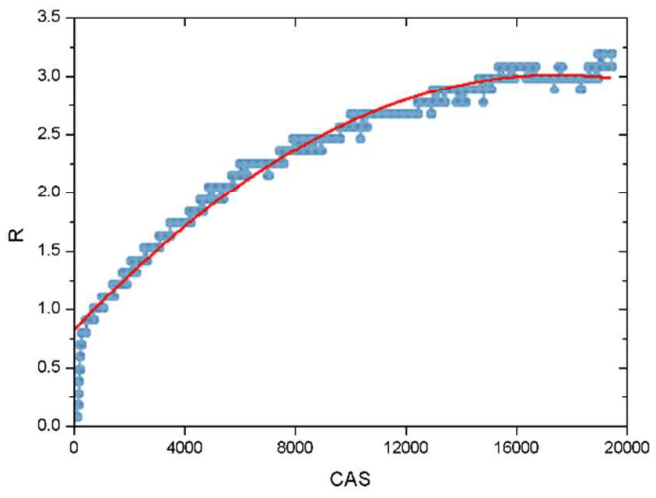

(A)

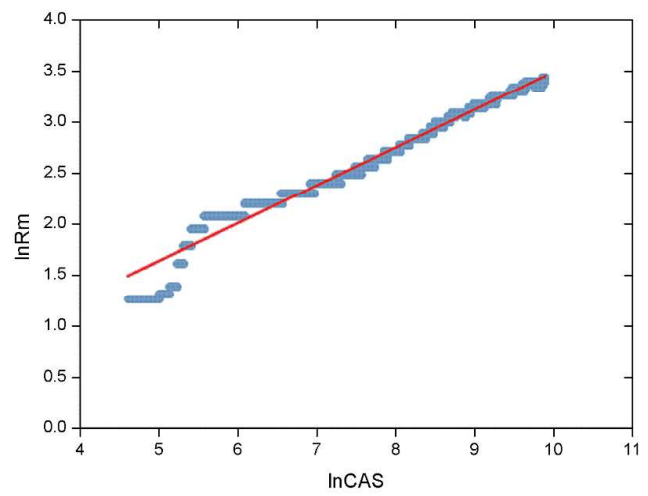

(B)

Fig.13 Relation between average grain size and CAS at different temperature and activation energy (A) different temperature (B) different activation energy

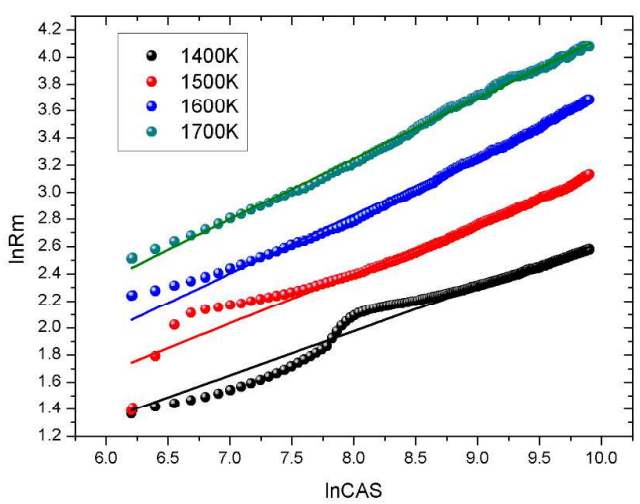

(A)

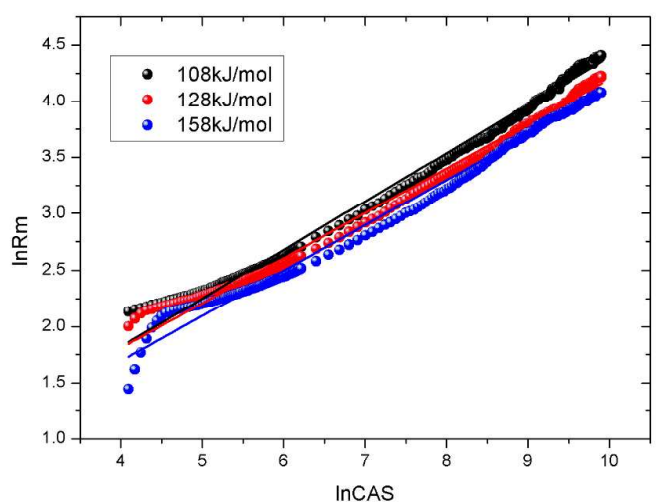

(B)

Fig.14 (A) Distribution of grains with different sides, (B) Growth kinetics of grains with different sides

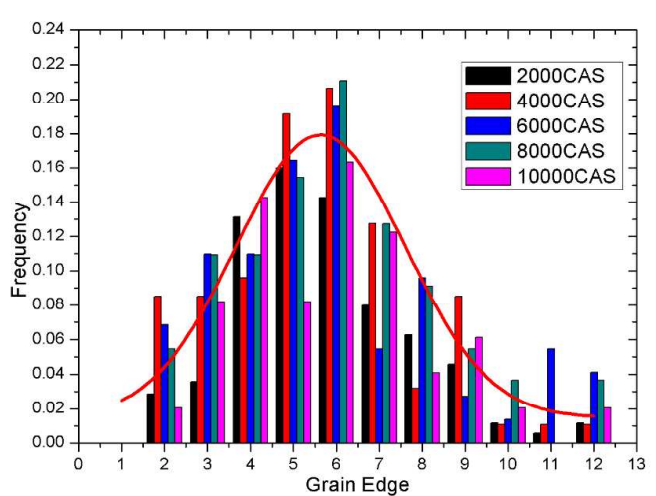

(A)

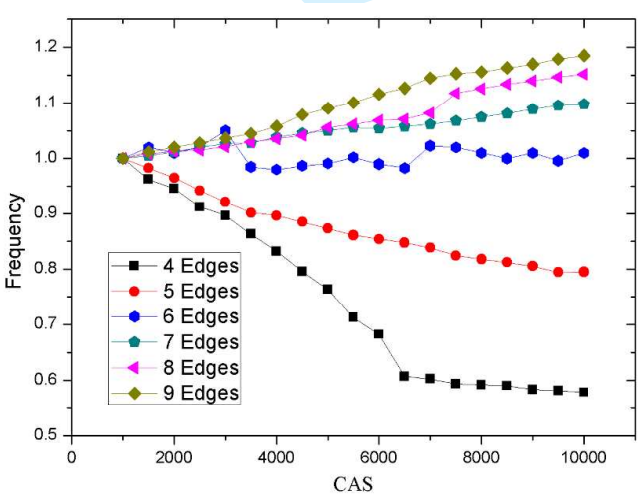

(B) 
Fig.15 Evolution of grain with different edges

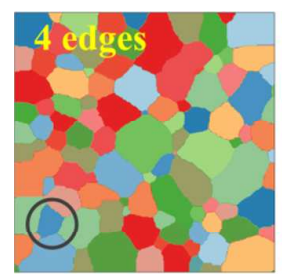

6000CAS

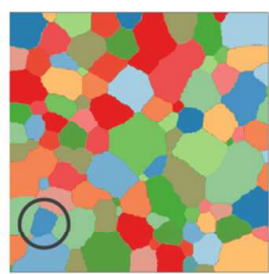

6500CAS

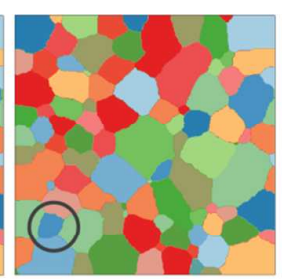

7000CAS

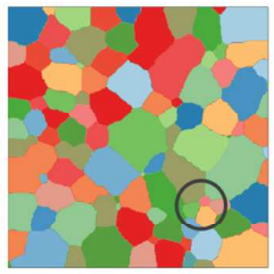

6800CAS

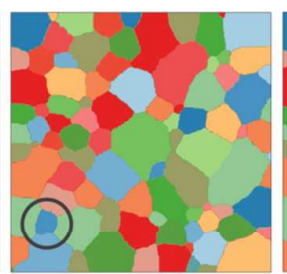

7500CAS

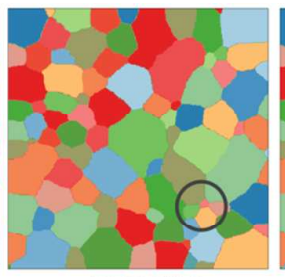

7200CAS

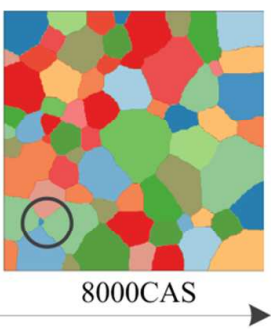

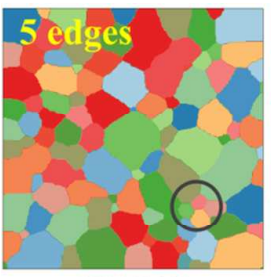

6000CAS

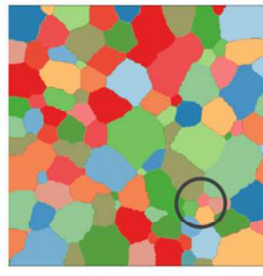

6400CAS

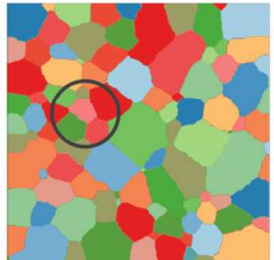

6800CAS

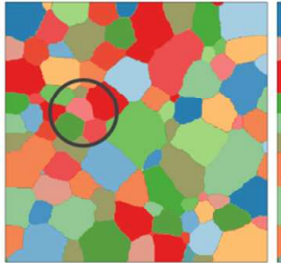

7200CAS

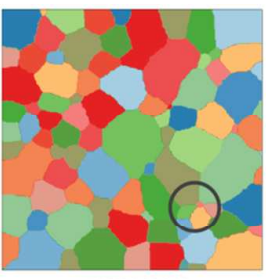

7600CAS

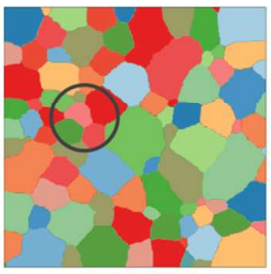

7600CAS

Fig.16 Evolution of grain with more than 6 edges

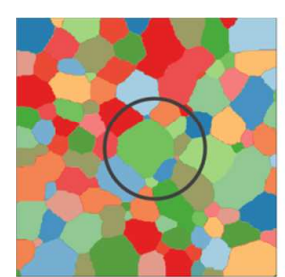

6000CAS

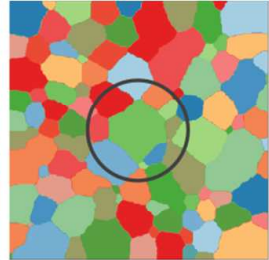

6500CAS

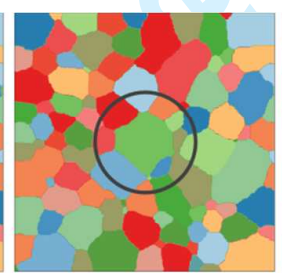

7000CAS

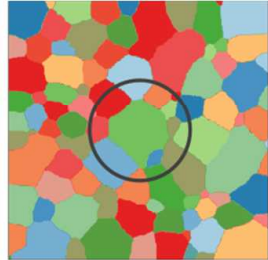

7500CAS

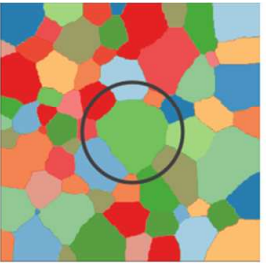

8000CAS 